\title{
Binary relevance model for activity recognition in home environment using ambient sensors
}

\begin{abstract}
One of the most important applications of the smart home environment is health monitoring and assistance by analysing activities of daily living and here Human Activity Recognition (HAR) plays a major role. The HAR problem, basically a temporal classification problem has been modelled in the past with various methods such as Bayesian Networks, Hidden Markov Model, Conditional Random Field, etc. Here, we propose the Binary Relevance Method of the multi- label classification to tackle the multi-resident activity recognition problem on real world dataset. Through the results obtained by the evaluation metrics namely accuracy, precision and hamming loss, it can be inferred that the model not only computes competitive results to previous works but also signifies the importance of the baseline Binary Relevance method to solve multi-label problems.
\end{abstract}

Keyword: Activity recognition; Binary relevance; Multi-label classification; Random forest; Smart home sensor 